\title{
Characterization of Germin-like Proteins (GLPs) and Their Expression in Response to Abiotic and Biotic Stresses in Cucumber
}

\author{
Liting Liao ${ }^{1,2,+}$, Zhaoyang $\mathrm{Hu}^{1,+}$, Shiqiang Liu ${ }^{1}\left(\mathbb{D}\right.$, Yingui Yang ${ }^{2, *}$ and Yong Zhou ${ }^{1,3, *(\mathbb{D})}$ \\ 1 College of Bioscience and Bioengineering, Jiangxi Agricultural University, Nanchang 330045, China; \\ 1lt18770156559@163.com (L.L.); huzhaoyang@jxau.edu.cn (Z.H.); 1sq_hn306@163.com (S.L.) \\ 2 College of Agronomy, Jiangxi Agricultural University, Nanchang 330045, China \\ 3 Key Laboratory of Crop Physiology, Ecology and Genetic Breeding, Ministry of Education, \\ Jiangxi Agricultural University, Nanchang 330045, China \\ * Correspondence: yangyingui@163.com (Y.Y.); yongzhou@jxau.edu.cn (Y.Z.) \\ + Contributed equally.
}

check for updates

Citation: Liao, L.; Hu, Z.; Liu, S.; Yang, Y.; Zhou, Y. Characterization of Germin-like Proteins (GLPs) and Their Expression in Response to Abiotic and Biotic Stresses in Cucumber. Horticulturae 2021, 7, 412. https: / / doi.org/10.3390/ horticulturae7100412

Academic Editors: Pirjo Mäkelä,

Mercè Llugany, Peter A. Roussos and Mumtaz Cheema

Received: 17 September 2021

Accepted: 15 October 2021

Published: 17 October 2021

Publisher's Note: MDPI stays neutral with regard to jurisdictional claims in published maps and institutional affiliations.

Copyright: (c) 2021 by the authors. Licensee MDPI, Basel, Switzerland. This article is an open access article distributed under the terms and conditions of the Creative Commons Attribution (CC BY) license (https:// creativecommons.org/licenses/by/ $4.0 /)$.

\begin{abstract}
Germins and germin-like proteins (GLPs) are glycoproteins closely associated with plant development and stress response in the plant kingdom. Here, we carried out genome-wide identification and expression analysis of the GLP gene family in cucumber to study their possible functions. A total of 38 GLP genes were identified in cucumber, which could be mapped to six out of the seven cucumber chromosomes. A phylogenetic analysis of the GLP members from cucumber, Arabidopsis and rice showed that these GLPs could be divided into six groups, and cucumber GLPs in the same group had highly similar conserved motif distribution and gene structure. Gene duplication analysis revealed that six cucumber GLP genes were located in the segmental duplication regions of cucumber chromosomes, while 14 genes were associated with tandem duplications. Tissue expression profiles of cucumber GLP genes showed that many genes were preferentially expressed in specific tissues. In addition, some cucumber GLP genes were differentially expressed under salt, drought and ABA treatments, as well as under DM inoculation. Our results provide important information for the functional identification of GLP genes in the growth, development and stress response of cucumber.
\end{abstract}

Keywords: cucumber; germin-like protein (GLP); abiotic stresses; downy mildew (DM); expression analysis

\section{Introduction}

Germin was first identified in wheat's embryos and labeled as a germination marker; it was subsequently characterized as a homohexamer glycoprotein with oxalate oxidase (OXO, EC 1.2.3.4) activity [1,2]. Proteins with similar amino acid sequences and structures to wheat germin are defined as germin-like proteins (GLPs) [3-5]. GLPs are a type of soluble glycoproteins with a "cupin" domain (PF00190) belonging to the "cupin superfamily" ubiquitously present in plants [6,7]. GLPs harbor a conserved $\beta$-sheet barrel domain in metal binding and play enzymatic and non-enzymatic roles by altering the region of the active site $[3,8,9]$. Most GLPs are stable oligomers, but they have enzyme activities most often in typically hexameric structures, being trimers of dimers form $[4,10]$. Besides OXO activity, various other enzymatic activities were found in GLPs, such as superoxide dismutase (SOD, EC 1.15.1.1) [10,11], ADP glucose pyrophosphatase/phosphodiesterase (AGPPase, EC 3.1.4.1) [12], and polyphenol oxidases (PPO) [13,14].

To date, many GLP genes have been isolated and functionally characterized in a variety of plant species, indicating their key roles in plant growth and development. For example, rice OsGLP1 (Os08g35760, also named as OsGLP8-14) is mainly found in green vegetative tissues and plays a determinant role in plant height [15]. OsGLP2-1 (Os02g29000) 
acts as an important regulator of seed dormancy in rice in the abscisic acid (ABA) and gibberellic acid (GA) signaling pathways [16]. Overexpression of rice OsRGLP1 (Os08g09080, also named as OsGLP8-11) in tomato resulted in morpho-physiological traits, including increased chlorophyll and relative water content, dwarfism, high density and diversity of trichomes [17]. In addition, GLPs are also related to the responses of plants to biotic and abiotic stress. For instance, among the eight tea plant $G L P s$, six and two genes were induced and repressed by insect herbivory, respectively [18]. The expression levels of many rice GLP genes were obviously altered under various biotic (brown plant hopper, Agrobacterium tumefaciens and fungal infestation) and abiotic (cold, salt, drought and anoxia) stress conditions [19]. Specifically, OsGLP8-14 was found to be involved in pathogen resistance [15], salt stress response [20] and acclimation to UV-B radiation [21]. In recent years, there has been increasing evidence demonstrating the crucial regulatory roles of GLP genes in plant disease resistance. For example, transgenic tobacco plants overexpressing Lilium regale LrGLP1 exhibited considerably enhanced resistance to Fusarium oxysporum infection [22]. Transgenic Solanum tuberosum and Medicago truncatula plants overexpressing OsRGLP1 also displayed higher resistance to Fusarium oxysporum [23,24]. Another rice GLP gene, OsGLP2-4 (Os02g32980), was found to confer resistance to fungal blast and bacterial blight in the jasmonic acid (JA)-dependent pathway [25]. Overexpression of sunflower HaGLP1 in Arabidopsis improved the resistance to fungal pathogens by promoting the accumulation of reactive oxygen species (ROS) [11]. Transgenic tobacco plants overexpressing soybean GmGLP10 gene displayed enhanced resistance to Sclerotinia sclerotiorum infection [26]. Cotton GhABP19 is also associated with the JA-mediated defense response to Verticillium dahliae and Fusarium oxysporum infection [27].

In recent years, genome-wide analysis of the GLP gene family has identified a large number of GLP genes in various plant species, such as Physcomitrella patens [28], soybean [29,30], tea plant [18], wheat [31], rice and Arabidopsis [6]. However, there is very limited information about the GLP genes in cucumber, an important vegetable crop frequently affected by diverse stresses. This study systematically analyzed the GLP family genes in cucumber. The findings may provide a theoretical basis for the functional analysis of GLP genes in cucumber and other plant species.

\section{Materials and Methods}

\subsection{Identification and Property Characterization of GLPs in Cucumber}

To identify all members of the GLP gene family in cucumber, the hidden Markov model (HMM) profile of the GLP feature domain (PF00190) was downloaded from the Pfam database (http:/ / pfam.xfam.org/, Pfam 34.0, 15 March 2021), and then the HMMER3 software was employed to identify the GLP genes in the cucumber (Chinese Long 9930) genome database (http:/ / cucurbitgenomics.org/organism/2, 15 March 2021). To ensure accuracy, all putative GLP protein sequences were subjected to Pfam (http:/ /Pfam.sanger.ac.uk/, 15 March 2021) and SMART (http:/ / smart.embl-heidelberg.de/, 15 March 2021) to verify the presence of the "cupin" domain (PF00190). The length of amino acids (aa), theoretical molecular weight (MW) and isoelectric point (pI) of the deduced CsGLP proteins were determined by the ProtParam program (http:/ / web.expasy.org/protparam, 15 March 2021). Subcellular localization prediction of the CsGLP proteins was carried out with the online tools including Plant-mPLoc (http:/ /www.csbio.sjtu.edu.cn/bioinf/plantmulti, 15 March 2021) and CELLO v.2.5 (http:/ / cello.life.nctu.edu.tw, 15 March 2021).

\subsection{Phylogenetic, Conserved Motif and Gene Structure Analyses}

To investigate the phylogenetic relationship between GLP proteins of cucumber and other plant species, the full-length GLP protein sequences of cucumber, Arabidopsis and rice were aligned using MAFFT with the default options, and a phylogenetic tree was constructed using the MEGA 7.0 software by the neighbor-joining (NJ) method with a bootstrap analysis of 1000 replications. To investigate the conserved motifs of GLP members from cucumber, the complete amino acid sequences of GLP proteins were analyzed 
using the online MEME tool (http: / / meme-suite.org/tools/meme, 15 March 2021). The parameters for the analysis were as follows: maximum number of motifs, 10; minimum motif width, 6; and maximum motif width, 50. The genomic library and coding sequence (CDS) information of cucumber were downloaded from the cucumber (Chinese Long) genome database (http:/ / cucurbitgenomics.org/organism/2, 15 March 2021). For gene structure analysis, the CDS and corresponding genomic sequences of cucumber GLP genes were retrieved, and the gene structure was analyzed by the Gene Structure Display Server (GSDS, http:/ /gsds.gao-lab.org/, 15 March 2021).

\subsection{Chromosomal Distribution and Gene Duplication Analysis}

The chromosomal locations of the CsGLP genes were analyzed by MapGene2Chrom (http://mg2c.iask.in/mg2c_v2.0/, 15 March 2021), a tool for the online reconstruction of the gene chromosome position map. Tandem and segmental duplication events were identified using the MCScanX software based on previously published criteria [32].

\subsection{RNA-Seq Expression Analysis of the CsGLP Genes}

The raw RNA-seq data from various cucumber tissues (roots, stems, leaves, flowers, ovaries and tendrils) and cucumber inoculated with downy mildew (DM, Pseudoperonospora cubensis) were download from the NCBI database (PRJNA80169 and SRP009350) and analyzed with StringTie according to our previous study [33]. For tempo-spatial expression analysis, a total of 10 tissues from Cucumis sativus var. satious line 9930 were sampled, including the root, stem, leaf, male and female flowers, base part of tendril, tendril, unexpanded ovary, expanded ovary under fertilization (7 days after flowering) and expanded ovary not fertilized (7 days after flowering) [34]. For DM inoculation expression analysis, leaf samples were collected from C. sativus cv. 'Vlaspik' at different time points after inoculation with $P$. cubensis [35]. The transcripts per kilobase million (TPM) values were $\log _{2}$ transformed using the TBtools software for generating heat map and cluster analysis [36].

\section{5. qRT-PCR Analysis of CsGLP Genes in Response to Abiotic Stress}

Seeds of the cucumber line 9930 were surface sterilized and germinated on wet filter paper in a growth chamber at $28{ }^{\circ} \mathrm{C}$ for 1 day. Then, the germinated seeds were transplanted onto poly trays containing peat, sand and pumice at a 1:1:1 ratio. The seedlings were subsequently transferred into hydroponic boxes filled with $1 / 2$ Hoagland nutrient solution in a greenhouse under 16-h light/8-h dark conditions once the cotyledon was fully unfolded. Seedlings at the three-leaf stage were treated with the salt, drought and ABA treatments by using $200 \mathrm{mM} \mathrm{NaCl}, 10 \%$ PEG-6000 (w/v) and $100 \mu \mathrm{M}$ ABA. Seedlings were subjected to salt and drought stress treatments by the addition of 10\% (w/v) PEG-6000 (Sigma-Aldrich, Shanghai, China) and $200 \mathrm{mM} \mathrm{NaCl}$ in 1/2 Hoagland nutrient solution, respectively. For ABA treatment, seedlings were treated with $100 \mu \mathrm{M}$ abscisic acid (ABA) as described previously [37]. Leaf samples were collected at 0, 6, 12 and $24 \mathrm{~h}$ after treatments and used for qRT-PCR analysis.

Total RNA was isolated using the Eastep Super Total RNA Extraction Kit (Promega, Madison, WI, USA), and cDNA synthesis was performed using the M-MLV reverse transcriptase (Invitrogen, USA) according to the protocols. qRT-PCR was performed with the Roche Lightcyler 480II PCR System using the TB Green Premix Ex TaqII Kit (TaKaRa, Dalian, China) under the guidelines, as previously described [32]. Three biological replicates were performed for each experiment. The CsAct3 gene was used as a control for qRT-PCR analysis with the $2^{-\Delta \Delta \mathrm{Ct}}$ relative quantitative method [32].

\section{Results}

\subsection{Identification of the CsGLP Genes in Cucumber}

In total, 38 candidate GLP genes were identified in the cucumber genome, which were assigned according to their chromosomal locations (Table 1). Then, the 38 deduced protein sequences were verified through scanning of Pfam and SMART. The results revealed that 
all the sequences had the cupin domain (PF00190), which is the typical configuration of GLP proteins, suggesting that they belong to the GLP gene family. The detailed information of each GLP member, including the locus ID, chromosomal location, length of CDS, gDNA and protein, is presented in Table 1. The gDNA of CsGLPs ranged from 564 bp (CsGLP1-2) to $3868 \mathrm{bp}$ (CsGLP7-4) and encoded the polypeptides with lengths ranging from 124 aa (CsGLP5-3) to 927 aa (CsGLP3-1). The MW ranged from 13.54 kDa (CsGLP5-3) to 112.93 $\mathrm{kDa}$ (CsGLP3-1), and the pI values from 5.16 (CsGLP1-3) to 9.43 (CsGLP7-3) (Table 1). Prediction of the subcellular localization of CsGLP proteins revealed that two CsGLPs were located in the nucleus, 12 CsGLPs were located in the vacuole, and 23 CsGLPs were located in the cell wall, while CsGLP3-2 was predicted to be located in both the cell wall and vacuole (Table 1 ).

\subsection{Phylogenetic Analysis of GLP Proteins from Cucumber and Other Plant Species}

To assess the evolutionary relationships of the 38 CsGLP proteins, a phylogenetic tree was constructed with MEGA 7.0 using the full-length GLP amino acid sequences from cucumber, Arabidopsis and rice. The phylogenetic tree demonstrated that these GLPs could be divided into six groups (Groups a-f), with Group a being the largest group in all three species (Figure 1). Group f (15 CsGLPs) and Group a (11 CsGLPs) comprised a larger number of cucumber members, while there were only five, three, two and two members from cucumber in Group e, Group b, Group c and Group d, respectively (Figure 1).

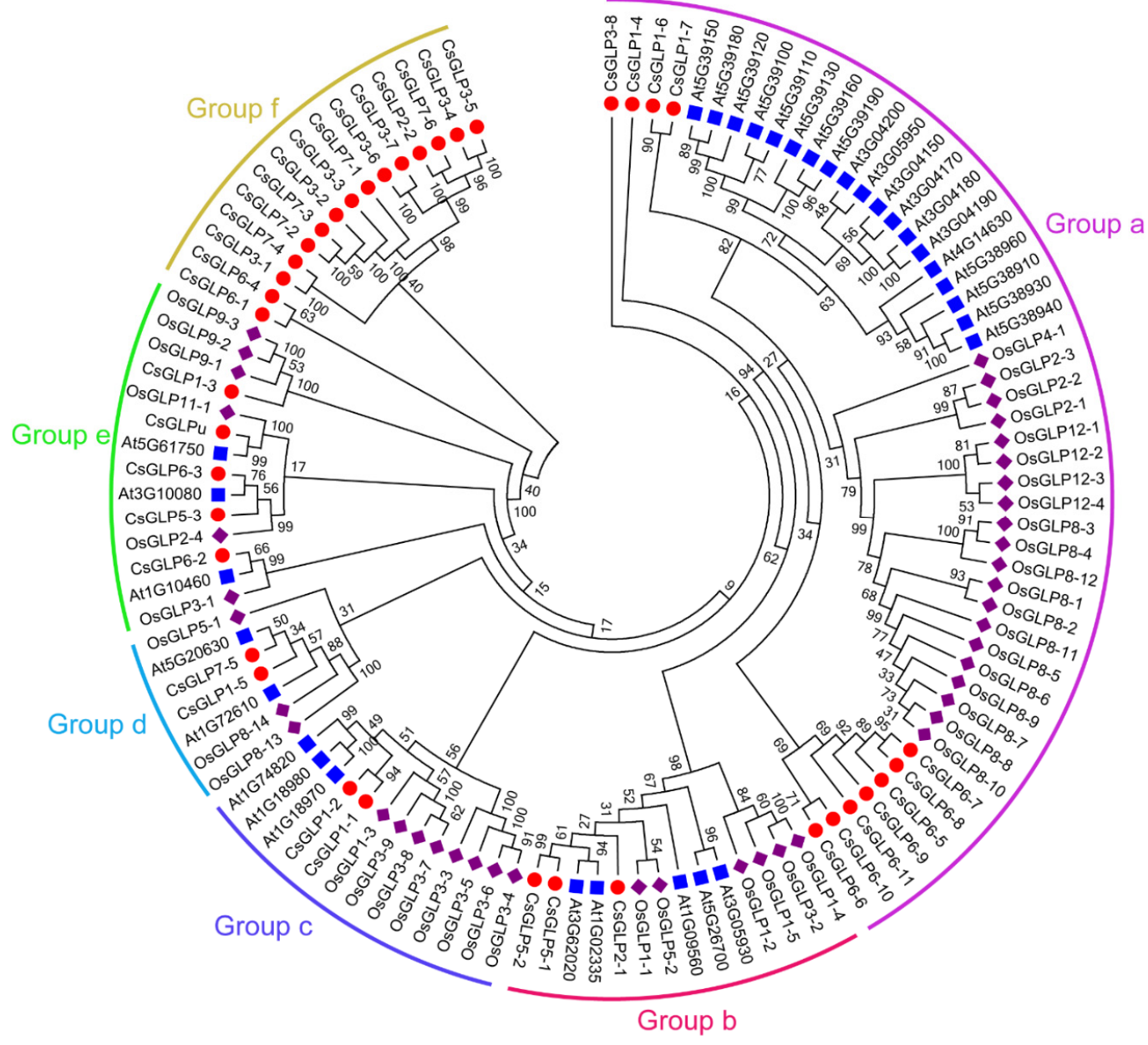

Figure 1. Phylogenetic relationships and subfamily designations of GLP proteins from cucumber, rice and Arabidopsis. The analysis involved 113 amino acid sequences_cucumber (38), rice (43) and Arabidopsis (32)—and their IDs are listed in Table S2. The phylogenetic tree of GLP proteins from cucumber, rice and Arabidopsis was constructed using the NJ method with 1000 bootstrap replicates, and GLP proteins in the tree were divided into six groups (Groups a-f). 
Table 1. Identification and characterization of GLP family genes in cucumber.

\begin{tabular}{|c|c|c|c|c|c|c|c|c|c|}
\hline \multirow[b]{2}{*}{ Gene } & \multirow[b]{2}{*}{ Locus } & \multirow[b]{2}{*}{ Chromosome } & \multirow[b]{2}{*}{ Chromosomal Position } & \multirow[b]{2}{*}{ gDNA (bp) } & \multirow[b]{2}{*}{ CDS (bp) } & \multicolumn{4}{|c|}{ Protein } \\
\hline & & & & & & Length (aa) & MW (Da) & $\mathrm{pI}$ & $\begin{array}{l}\text { Subcellular } \\
\text { Prediction }\end{array}$ \\
\hline CsGLP1-1 & Csa1G007890.1 & 1 & $1255048-1256300$ & 1253 & 666 & 221 & $23,666.44$ & 7.73 & Cell wall \\
\hline CsGLP1-2 & Csa1G007900.1 & 1 & $1258859-1259500$ & 642 & 564 & 213 & $22,984.84$ & 7.81 & Cell wall \\
\hline CsGLP1-3 & Csa1G166250.1 & 1 & $10344063-10344626$ & 564 & 675 & 187 & $19,869.54$ & 5.16 & Cell wall \\
\hline CsGLP1-4 & Csa1G537570.1 & 1 & 19610111-19612225 & 2115 & 672 & 217 & $23,108.48$ & 5.79 & Cell wall \\
\hline CsGLP1-5 & Csa1G596420.1 & 1 & $22537064-22539018$ & 1955 & 642 & 215 & $22,364.19$ & 6.81 & Cell wall \\
\hline CsGLP1-6 & Csa1G662790.1 & 1 & $26758087-26759600$ & 1514 & 648 & 224 & $23,991.83$ & 7.82 & Cell wall \\
\hline CsGLP1-7 & Csa1G662810.1 & 1 & 26786634-26788514 & 1881 & 654 & 223 & $24,103.81$ & 6.82 & Cell wall \\
\hline CsGLP2-1 & Csa2G035370.1 & 2 & $3544666-3546134$ & 1469 & 654 & 217 & $22,892.41$ & 9.30 & Cell wall \\
\hline CsGLP2-2 & Csa2G174130.1 & 2 & $9968726-9970593$ & 1868 & 1464 & 487 & $55,464.56$ & 8.83 & Vacuole \\
\hline CsGLP3-1 & Csa3G146460.1 & 3 & 9816841-9820025 & 3185 & 1440 & 927 & $112,934.56$ & 8.43 & Nucleus \\
\hline CsGLP3-2 & Csa3G218160.1 & 3 & $14499431-14500893$ & 1463 & 1023 & 340 & $37,226.71$ & 5.83 & Cell wall, Vacuole \\
\hline CsGLP3-3 & Csa3G218170.1 & 3 & $14512631-14514977$ & 2347 & 1071 & 356 & $38,684.19$ & 5.19 & Vacuole \\
\hline CsGLP3-5 & Csa3G386310.1 & 3 & 18896546-18898442 & 1897 & 1485 & 494 & $56,770.06$ & 8.60 & Vacuole \\
\hline CsGLP3-6 & Csa3G386810.1 & 3 & 18901334-18903344 & 2011 & 447 & 479 & $54,416.18$ & 5.71 & Vacuole \\
\hline CsGLP3-7 & Csa3G386820.1 & 3 & $18920239-18921873$ & 1635 & 1047 & 348 & $40,180.34$ & 5.51 & Vacuole \\
\hline CsGLP3-8 & Csa3G644800.1 & 3 & $25260870-25261483$ & 614 & 1506 & 148 & $16,322.28$ & 6.63 & Cell wall \\
\hline CsGLP5-1 & Csa5G128780.1 & 5 & 3162233-3164925 & 2693 & 675 & 233 & $25,061.75$ & 6.06 & Cell wall \\
\hline CsGLP5-2 & Csa5G129280.1 & 5 & $3171528-3174320$ & 2793 & 702 & 224 & $23,714.30$ & 6.96 & Cell wall \\
\hline CsGLP5-3 & Csa5G614670.1 & 5 & $24218204-24218890$ & 687 & 375 & 124 & $13,540.72$ & 6.51 & Cell wall \\
\hline CsGLP6-1 & Csa6G290870.1 & 6 & $14050717-14053164$ & 2448 & 1527 & 508 & $58,057.85$ & 5.74 & Vacuole \\
\hline CsGLP6-2 & Csa6G404270.1 & 6 & 18237396-18238128 & 733 & 663 & 224 & $24,344.08$ & 8.37 & Cell wall \\
\hline CsGLP6-3 & Csa6G452110.1 & 6 & $21556627-21557475$ & 849 & 657 & 207 & $22,359.95$ & 8.77 & Cell wall \\
\hline CsGLP6-4 & Csa6G502040.1 & 6 & $25250904-25253534$ & 2631 & 648 & 661 & $78,191.37$ & 6.23 & Nucleus \\
\hline CsGLP6-5 & Csa6G525540.1 & 6 & 28560994-28561809 & 816 & 648 & 219 & $23,425.11$ & 7.80 & Cell wall \\
\hline CsGLP6-6 & Csa6G525550.1 & 6 & $28563130-28563904$ & 775 & 651 & 216 & $22,819.23$ & 6.69 & Cell wall \\
\hline
\end{tabular}


Table 1. Cont.

\begin{tabular}{|c|c|c|c|c|c|c|c|c|c|}
\hline \multirow[b]{2}{*}{ Gene } & \multirow[b]{2}{*}{ Locus } & \multirow[b]{2}{*}{ Chromosome } & \multirow[b]{2}{*}{ Chromosomal Position } & \multirow[b]{2}{*}{ gDNA (bp) } & \multirow[b]{2}{*}{ CDS (bp) } & \multicolumn{4}{|c|}{ Protein } \\
\hline & & & & & & Length (aa) & MW (Da) & pI & $\begin{array}{l}\text { Subcellular } \\
\text { Prediction }\end{array}$ \\
\hline CsGLP6-7 & Csa6G525580.1 & 6 & $28567264-28568054$ & 791 & 660 & 215 & $22,597.97$ & 5.47 & Cell wall \\
\hline CsGLP6-8 & Csa6G525590.1 & 6 & 28570081-28570968 & 888 & 651 & 215 & $22,664.07$ & 5.84 & Cell wall \\
\hline CsGLP6-9 & Csa6G525600.1 & 6 & 28572339-28573248 & 910 & 624 & 216 & $23,153.73$ & 7.85 & Cell wall \\
\hline CsGLP6-10 & Csa6G525610.1 & 6 & $28574435-28575220$ & 786 & 1986 & 218 & $22,902.41$ & 6.26 & Cell wall \\
\hline CsGLP6-11 & Csa6G525620.1 & 6 & 28576583-28577614 & 1032 & 675 & 220 & $23,148.55$ & 6.95 & Cell wall \\
\hline CsGLP7-1 & Csa7G281380.1 & 7 & 9939437-9941121 & 1685 & 1071 & 356 & $38,300.97$ & 5.82 & Vacuole \\
\hline CsGLP7-2 & Csa7G337100.1 & 7 & 12086664-12088637 & 1974 & 708 & 339 & $37,153.37$ & 5.37 & Vacuole \\
\hline CsGLP7-3 & Csa7G368140.1 & 7 & $12991068-12992188$ & 1121 & 1431 & 235 & $25,649.72$ & 9.43 & Vacuole \\
\hline CsGLP7-4 & Csa7G380130.1 & 7 & 14118117-14121984 & 3868 & 1458 & 485 & $55,928.57$ & 6.94 & Vacuole \\
\hline CsGLP7-5 & Csa7G450510.1 & 7 & 18555157-18556979 & 1823 & 627 & 208 & $21,395.90$ & 6.39 & Cell wall \\
\hline CsGLP7-6 & Csa7G452090.1 & 7 & 18921704-18923747 & 2044 & 1020 & 476 & $54,181.04$ & 7.69 & Vacuole \\
\hline CsGLPu & CsaUNG024810 & Scaffold000221 & $4018-4951$ & 934 & 690 & 229 & $25,058.56$ & 7.00 & Cell wall \\
\hline
\end{tabular}




\subsection{Conserved Motif Analysis of Cucumber GLP Proteins}

The conserved motifs of the 38 cucumber GLP proteins were analyzed using the MEME online software, resulting in the prediction of 10 conserved motifs (Figure 2). Among them, motif 1 was present in all CsGLPs except for CsGLP3-8, and motif 2 and motif 3 were also present in most CsGLP proteins. Most CsGLPs in the same group harbored common motifs. For example, motif 4 and motif 5 were only observed in CsGLPs from Groups a-e, while motif 7, motif 8 and motif 9 were exclusively present in the CsGLPs of Group $\mathrm{f}$ (Figure 2B). In addition, motif 6 was widely present in CsGLPs from Groups a-e, as well as in CsGLP7-1 from Group $\mathrm{f}$. It is worth noting that CsGLP3-1, CsGLP6-1, CsGLP6-4, CsGLP7-3 and CsGLP7-4 from Group f harbored two motif 1, while CsGLP3-7 and CsGLP6-10 contained an additional motif 9 and motif 10, respectively (Figure 2B).

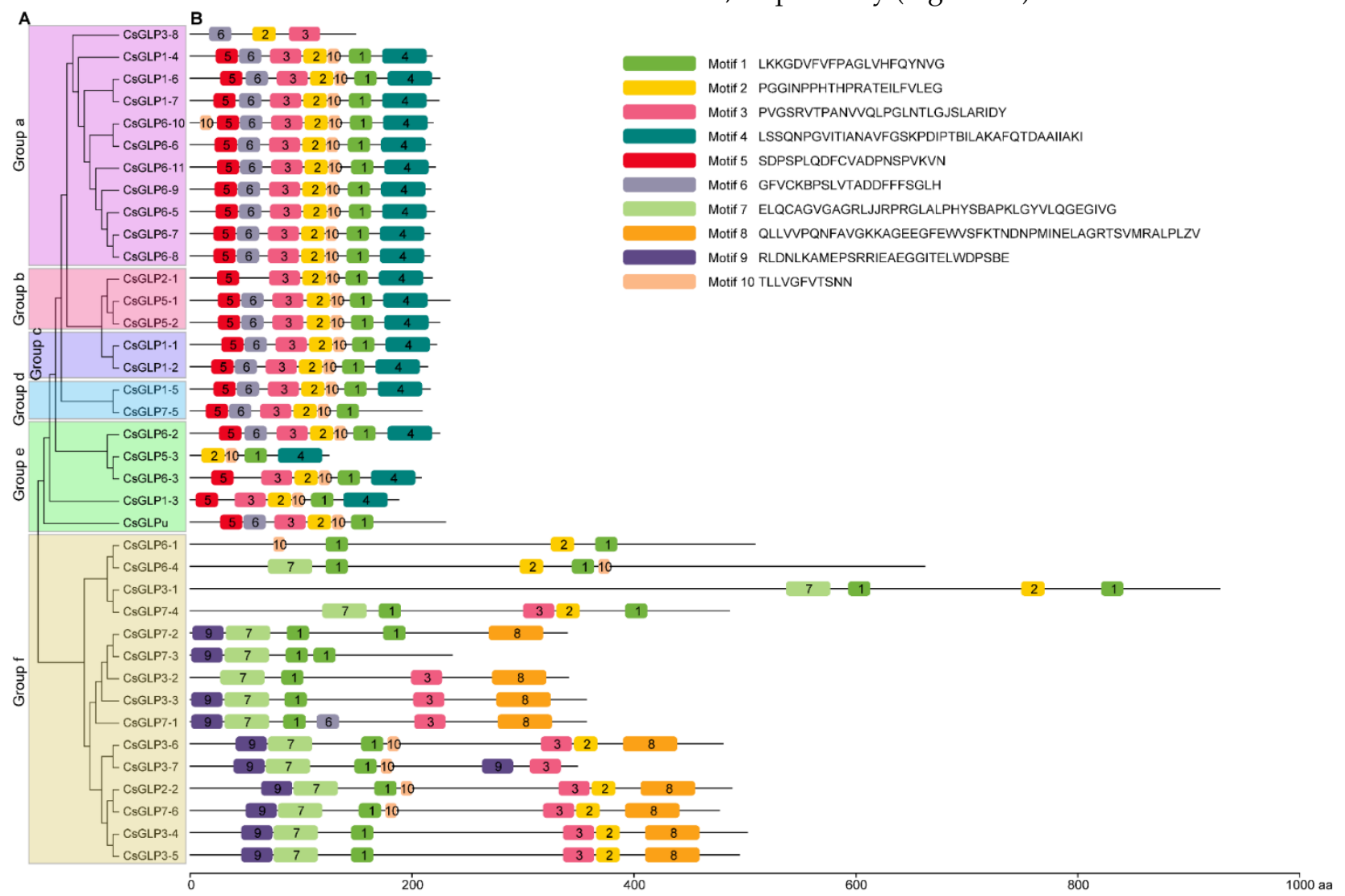

Figure 2. Phylogenetic relationship (A) and conserved motif arrangement (B) of cucumber GLP family members. (A) The phylogenetic tree of CsGLP proteins was created using the NJ method with 1000 bootstrap replicates, and the CsGLP proteins can be divided into six groups (Groups a-f). (B) Distribution of 10 conserved motifs among CsGLP proteins identified by MEME, and their amino acid sequences are shown at the right edge.

\subsection{Structure Analysis of CsGLP Genes}

Gene structure diversity is a possible mechanism for the evolution of multiple gene families [38]. We then analyzed the structural diversity of CsGLP genes with the GSDS tool. As shown in Figure 3, the intron number of the CsGLP genes ranged from 0 to 5, and nearly half of the CsGLP genes (18/38) were transcriptionally encoded by two exons and one intron. Specifically, seven CsGLP genes (CsGLP1-1, CsGLP1-2, CsGLP1-3, CsGLP5-3, CsGLP6-2, CsGLP6-3 and CsGLP7-5), which were clustered in Groups c-e, were found to be intronless (Figure 3). In addition, nearly all CsGLP genes in Group a and Group b harbored only one intron, except for CsGLP3-8, which possessed three introns. 


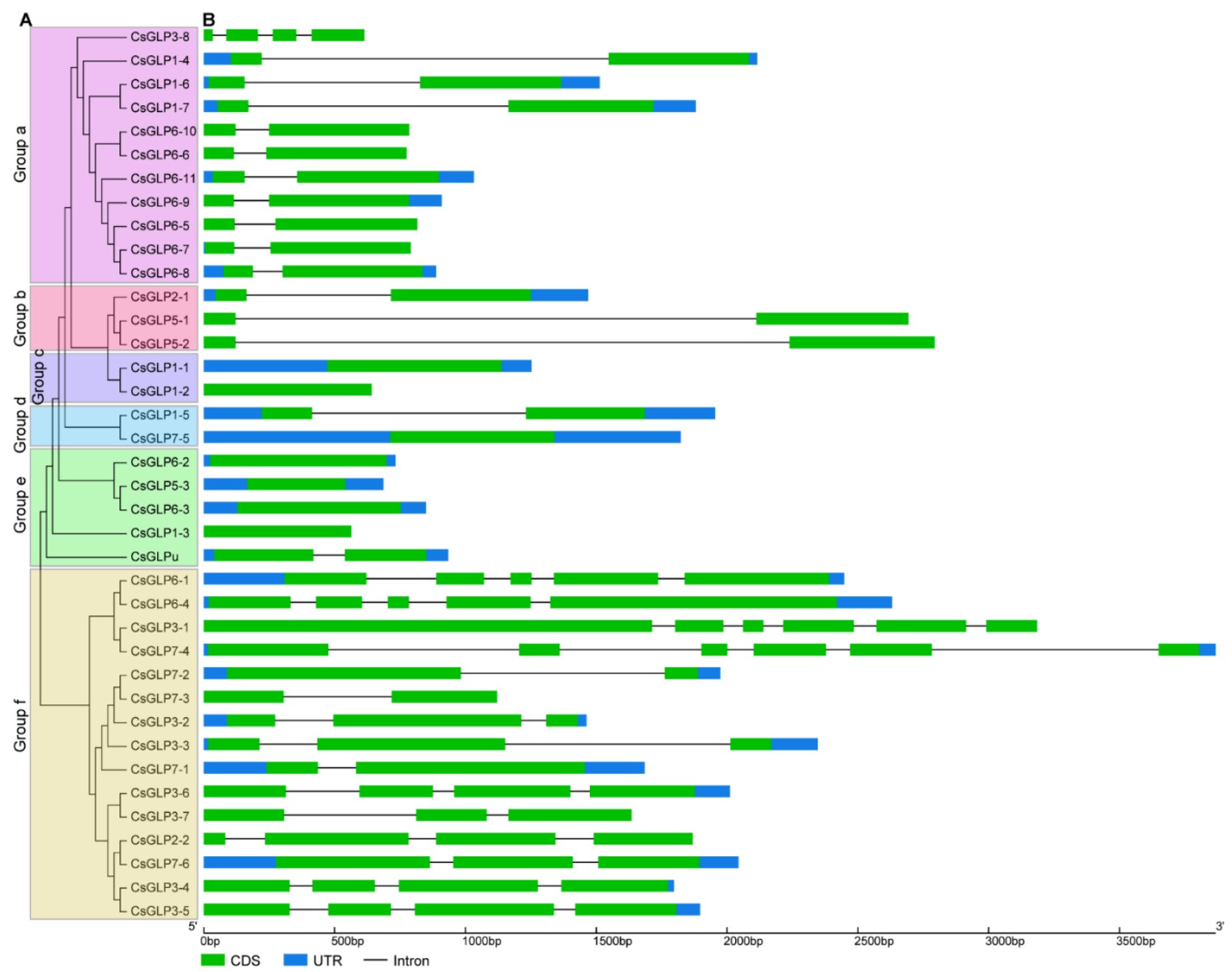

Figure 3. Phylogenetic relationship (A) and gene structure (B) of cucumber GLP family members. (A) The phylogenetic tree of CsGLP proteins was created using the NJ method with 1000 bootstrap replicates, and CsGLP proteins were divided into six groups (Groups a-f). (B) Gene structures of CsGLP genes analyzed by GSDS. Green box: CDS; Blue box: UTR; Black line: intron.

\subsection{Chromosomal Location and Gene Duplication of CsGLP genes}

To determine the chromosomal locations of CsGLP genes, a chromosomal map was constructed (Figure 4). A total of 37 CsGLP genes were distributed on six of seven cucumber chromosomes (except for chromosome 4), and one CsGLP gene was assigned to the scaffold000221. Amongst them, 11 CsGLP genes were found on chromosome 6, eight genes on chromosome 3 , seven genes on chromosome 1 , six genes on chromosome 7 , three genes on chromosome 5 and only two genes on chromosome 2 (Figure 4). We also determined the duplication events of the CsGLP genes. The results showed that three pairs of CsGLP genes underwent segmental duplication, including CsGLP1-4/CsGLP6-6, CsGLP1-5/CsGLP7-5 and CsGLP2-2/CsGLP3-4. In addition, five tandem duplication events involving 14 CsGLP genes were also observed (Figure 4). 


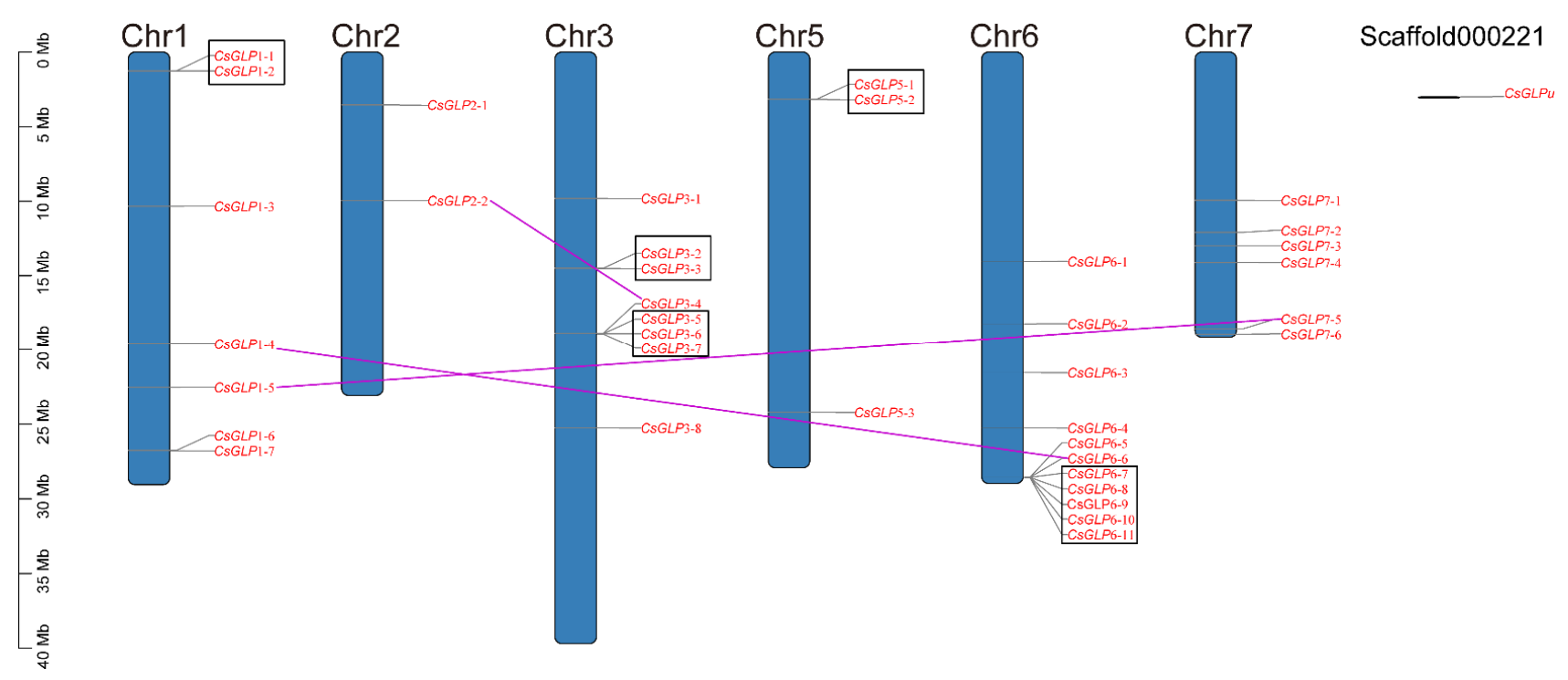

Figure 4. Chromosomal distributions and gene duplications of GLP genes in cucumber genome. The chromosome numbers are shown at the top of each chromosome.

\subsection{Expression Profiles of CsGLP Genes in Various Tissues}

To gain insights into the possible functions of the CsGLP genes, their temporal and spatial transcription patterns in various tissues were analyzed based on the published transcriptome data (Figure 5). A total of 24 CsGLP genes were found to be expressed in at least one of the tested tissues. Amongst them, some CsGLP genes were exclusively expressed in certain tissues, while other genes showed constitutive expression in different tissues. For example, CsGLP3-5 and CsGLP3-6 were specifically expressed in stems and tendrils, respectively (Figure 5). CsGLP6-1 and CsGLP6-4 showed preferential expression in the male flower, while CsGLP1-5 exhibited the highest transcriptional level in leaves. Several CsGLP genes, such as CsGLP1-6, CsGLP1-7, CsGLP5-2, CsGLP5-3, CsGLP6-3, CsGLP6-7, CsGLP6-8, CsGLP6-11 and CsGLPu, showed higher expression levels in roots than in other tissues (Figure 5), suggesting their specific roles in root development. In addition, CsGLP3-2 and CsGLP3-3 exhibited remarkable accumulation of transcripts in unfertilized and fertilized ovaries but not in the unexpanded ovary, and some other CsGLP genes including CsGLP1-1, CsGLP1-4, CsGLP1-5, CsGLP1-6, CsGLP2-1, CsGLP5-3, CsGLP7-1, CsGLP7-5 and CsGLP7-6 were also differentially transcribed in ovaries (Figure 5).

\subsection{Expression Patterns of CsGLP Genes in Response to DM Treatment}

To study the possible roles of CsGLP genes in response to biotic stress, the expression levels of CsGLP genes under downy mildew (DM) inoculation were determined based on the available RNA-seq data [35]. Compared with those in the mock control, a total of nine CsGLP genes (CsGLP1-1, CsGLP1-6, CsGLP2-1, CsGLP5-3, CsGLP6-7, CsGLP6-8, CsGLP69, CsGLP6-11 and CsGLP7-5) displayed up-regulated expression under DM inoculation (Figure 6). In particular, CsGLP2-1 and CsGLP5-3 were significantly up-regulated at the earlier time point of infection (1 dpi) and also showed increases in transcription at the later time points. However, the other seven CsGLP genes were significantly up-regulated at the later time points, especially at $6 \mathrm{dpi}$ and $8 \mathrm{dpi}$ (Figure 6). In addition, the expression of five CsGLP genes (CsGLP1-5, Cs GLP5-2, CsGLP6-2, CsGLP7-1 and CsGLP7-2) was significantly decreased by DM treatment compared with the control (Figure 6). The results suggested that the CsGLP genes might play key roles in the response of cucumber to DM infection. 


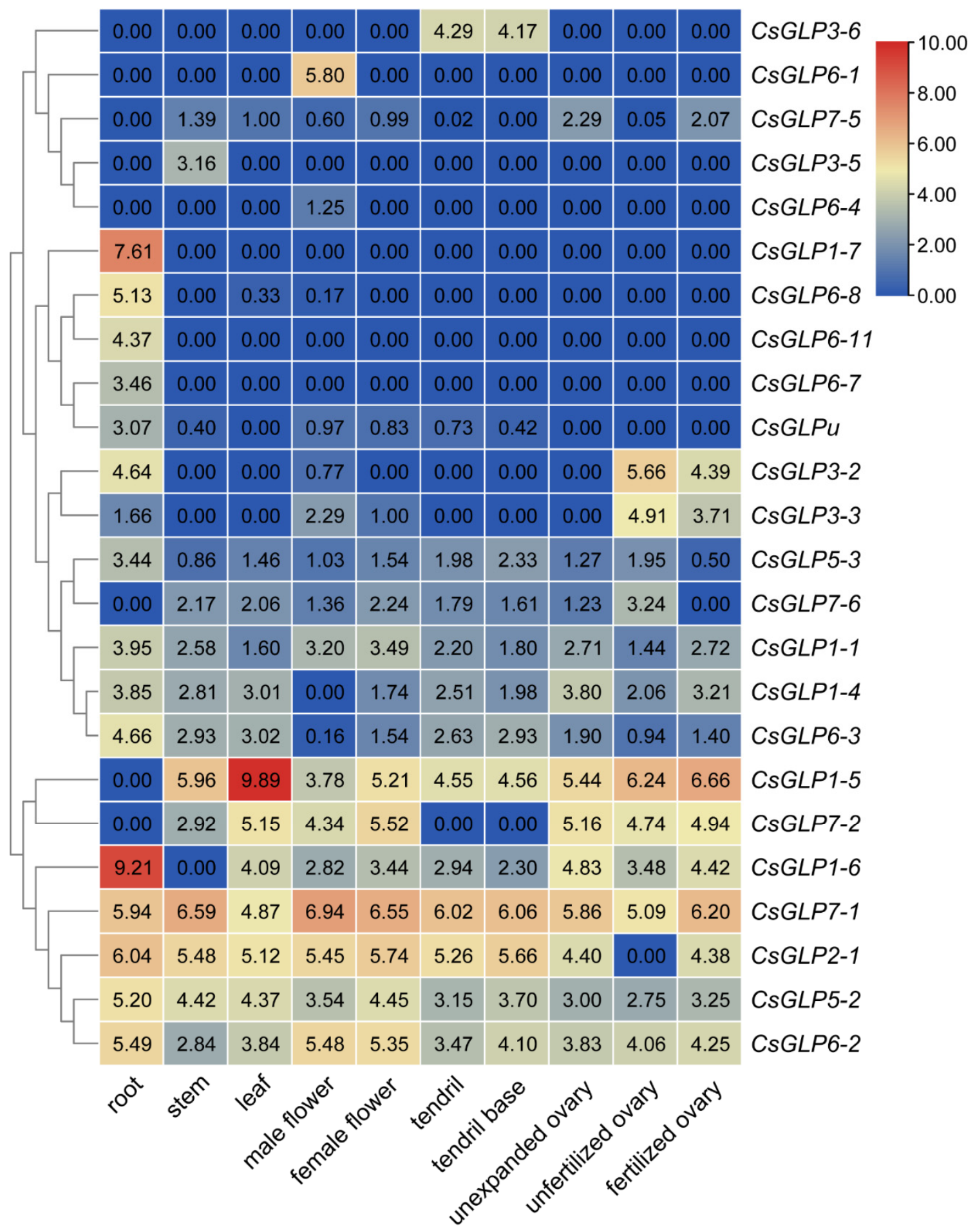

Figure 5. Expression levels of CsGLP genes in different cucumber tissues. 


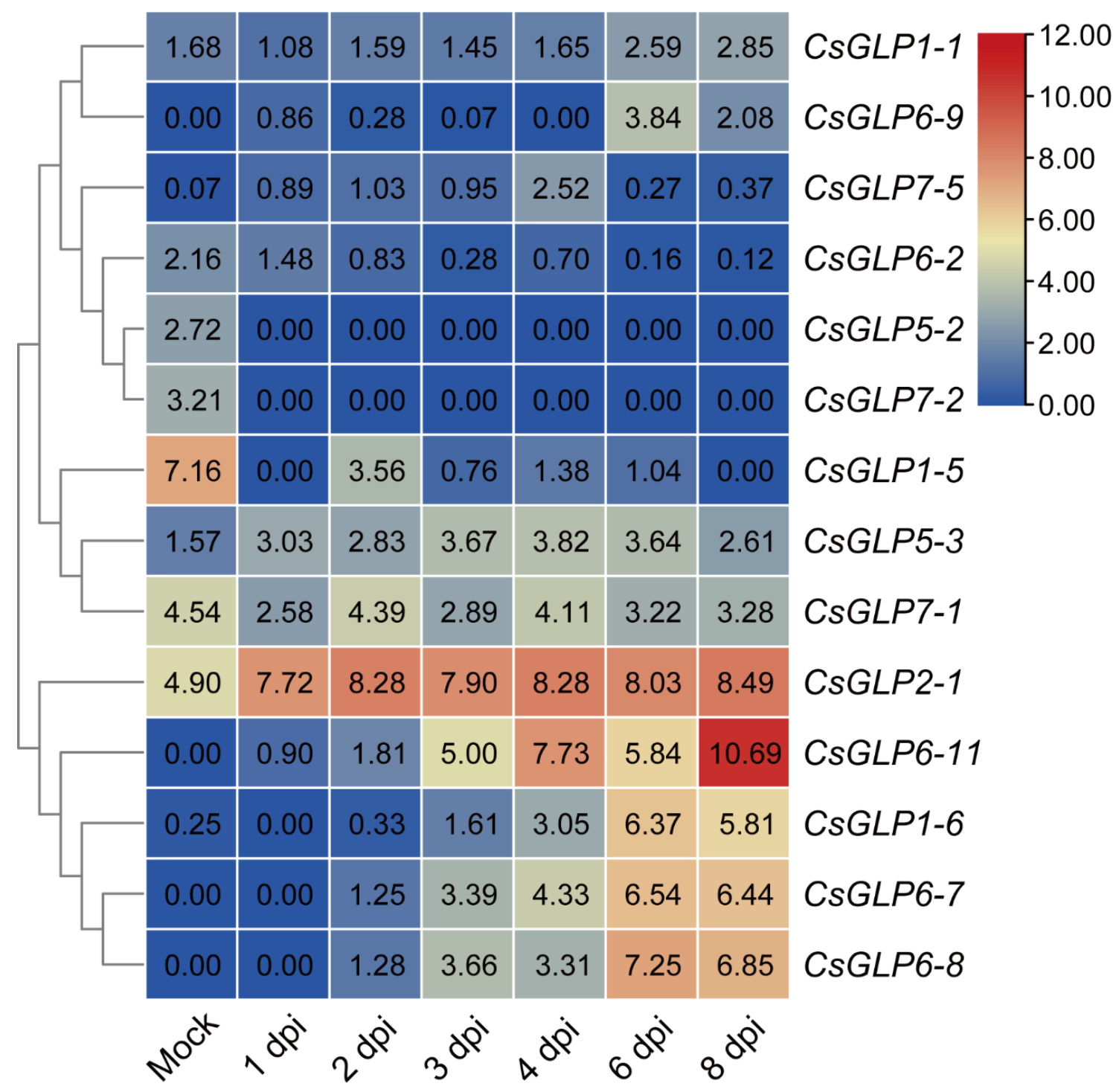

Figure 6. Expression patterns of CsGLP genes in response to inoculation with downy mildew for 1 to 8 days post inoculation (dpi). Mock, mock-inoculated sample collected at $1 \mathrm{dpi}$.

\subsection{Expression Patterns of Selected CsGLP Genes in Response to Salt, Drought and $A B A$ Treatments}

The expression patterns of five selected CsGLP genes in response to salt, drought and ABA treatments were determined by qRT-PCR. Under salt treatment, the transcription levels of CsGLP1-6, CsGLP5-2 and CsGLP7-1 were obviously increased at $12 \mathrm{~h}$, followed by a decrease at $24 \mathrm{~h}$. And the transcription of CsGLP1-5 was observably down-regulated, while the transcription of CsGLP2-1 was dramatically up-regulated after salt treatment at all time points (Figure 7A). Under drought treatment, CsGLP1-5 and CsGLP1-6 exhibited a decrease in expression at $6 \mathrm{~h}$, followed by increases at the subsequent time points. The transcription of CsGLP1-5, CsGLP1-6, CsGLP2-1 and CsGLP7-1 was induced and reached the peak at $12 \mathrm{~h}$ or $24 \mathrm{~h}$, while that of CsGLP5-2 was significantly down-regulated after drought treatment at all time points (Figure 7B). Under ABA treatment, the expression of CsGLP1-5 and CSGLP2-1 was significantly induced and reached the highest level at $12 \mathrm{~h}$, while that of CsGLP1-6, CsGLP5-2 and CsGLP7-1 displayed significant decreases at all time points (Figure 7C). The results indicated that these CsGLP genes are differentially regulated in response to salt, drought and ABA treatments. 

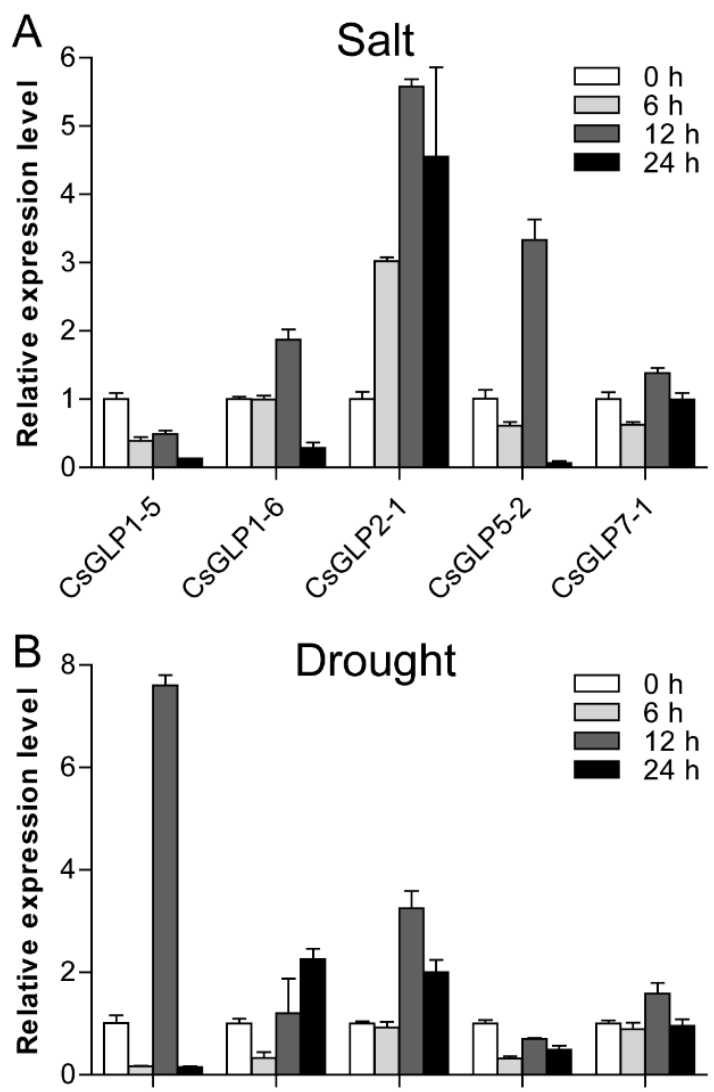

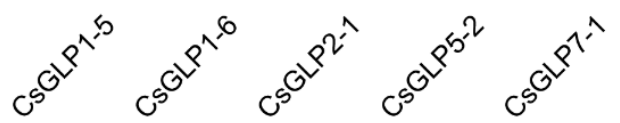

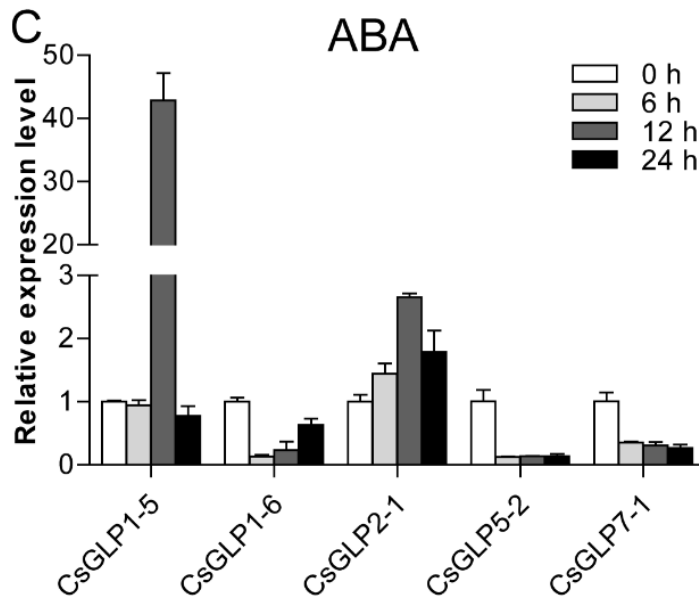

Figure 7. qRT-PCR analysis of expression patterns of five selected CsGLP genes in responses to salt (A), drought (B) and ABA (C) treatments.

\section{Discussion}

GLPs are a class of glycoproteins encoded in a multigene family and widely present in many plants. For example, it has been reported that there are 32 GLP genes in Arabidopsis [6] 43 in rice [6,39], 69 in soybean [30], 77 in P. patens [28] and 258 in wheat [31]. In the present study, a total of 38 GLP family members were identified and characterized in cucumber (Table 1). The number of cucumber GLP genes is comparable to that of rice and Arabidopsis, but much larger than that of the tea plant, which only has eight GLP genes [18]. The number of GLP genes shows no proportional variations along with the genome size of the above-mentioned plants, suggesting that gene duplication plays an 
important role in the expansion of GLP genes for diversification. In this study, three pairs of CsGLP genes were involved in segmental duplication, and 14 CsGLP genes constituted tandem duplication events (Figure 4).

Consistent with the results in rice and Arabidopsis [6], the 38 CsGLPs were clustered into six groups (Groups a-f), among which the largest number of members from cucumber belonged to Group $\mathrm{f}$ (Figure 1). We further examined the conserved motif distribution of cucumber GLP proteins according to evolutionary relationships. In total, 10 conserved motifs were identified, whose distributions exhibited strong evolutionary conservation (Figure 2), indicating that GLP proteins in the same group might have similar functions. Gene structure can also provide important insights into the evolutionary relationships among gene families [38]. In our results, most CsGLP members were found to contain only one intron (Figure 3), which is in accordance with the results in previous studies $[6,11]$. Moreover, the genes in the same group tended to exhibit similar intron numbers and CDS lengths (Figure 3), indicating that their functions may be similar. In addition, about $18 \%$ CsGLP genes (7 out of 38) were found to contain no intron at all, which was also observed in soybean [30], rice and Arabidopsis [6].

Previous studies have shown that GLPs play various roles in many physiological processes, such as plant height [15], fiber development [40] and seed dormancy [16]. We then investigated the tissue expression patterns of CsGLP genes based on the transcriptome data from different tissues of cucumber [34]. As shown in Figure 5, many CsGLP genes showed preferential expression in specific tissues. For example, predominant expression levels of some CsGLP genes were found in roots (CsGLP1-6, CsGLP1-7, CsGLP6-7, CsGLP6-8, CsGLP6-11 and CsGLPu), stems (CsGLP3-5), leaves (CsGLP1-5), male flowers (CsGLP6-1), and tendrils (CSGLP3-6), indicating that they play essential roles in these tissues. In addition, some CsGLP genes exhibited differential expression in different developmental stages of ovaries (Figure 5), suggesting their possible regulatory roles in the ovary development of cucumber.

In cucumber, DM caused by P. cubensis is a serious disease that results in severe damage to the production of cucumber around the world [41,42]. We then analyzed the expression of CsGLP genes to explore their possible roles in response to DM infection. As a result, nine and five CsGLP genes were up-regulated and down-regulated in response to the inoculation of DM (Figure 6), implying their possible roles in response to DM infection. It should be noted that CsGLP6-7, CsGLP6-8, CsGLP6-9 and CsGLP6-11 were significantly induced under DM inoculation, particularly at the later stage of infection (Figure 6), and they were found to be tandemly duplicated (Figure 4). Similar results were also reported in other plants. For example, a total of 12 rice OsGLP genes clustered in chromosome 8 confer resistance against two fungal pathogens: Magnaporthe oryzae and Rhizoctonia solani [43]. In wheat, most of the Blumeria graminis f. sp. tritici (Bgt) resistance-related TaGLP genes are also repeated in large tandem on the $4 \mathrm{~A}, 4 \mathrm{~B}$ and $4 \mathrm{D}$ chromosomes [31].

Plant GLPs also play key roles in the regulation of the abiotic stress response. Some GLPs were found to have SOD activity and could protect plants from antioxidant stress by converting harmful ROS into $\mathrm{H}_{2} \mathrm{O}_{2}$. For example, overexpression of sunflower $\mathrm{HaGLP1}$ in Arabidopsis resulted in higher resistance to fungal pathogens by promoting ROS accumulation [11]. Craterostigma plantagineum CPGLP1 is induced by dehydration and ABA, shows SOD activity and is involved in ROS metabolism and dehydration-related cell wall folding during desiccation [44]. Cotton GhGLP2 can also confer tolerance to oxidative stress in transgenic Arabidopsis plants by eliminating excess ROS due to its SOD activity [10]. In the present study, we observed that the five selected CsGLP genes showed differential expression in response to salt, drought and ABA treatments (Figure 7). Notably, CsGLP2-1 was significantly induced by the three treatments, and CsGLP1-6 and CSGLP7-1 were up-regulated under salt and drought stress but down-regulated under ABA treatment, suggesting their positive roles in regulating the salt and drought stress response in the ABA-dependent signal pathways. In addition, CsGLP1-5 and CsGLP5-2 displayed an oppo- 
site expression pattern under salt and drought stress (Figure 7A,B), suggesting that they might play divergent regulatory roles in response to the two stresses.

\section{Conclusions}

In this study, we performed a comprehensive characterization of the GLP genes in cucumber through systematic analysis of their phylogenetic relationships, conserved motifs, gene structures and expression profiles in response to salt, drought and ABA treatments, as well as under DM inoculation. A total of 38 GLP family genes were identified in the cucumber genome, and both segmental and tandem duplications are main mechanisms for GLP gene expansion in cucumber. Based on their phylogenetic relationship to the corresponding members in Arabidopsis and rice, CsGLPs could be classified into six groups (Group a-f), with highly similar conserved motif distribution and exon-intron structure within the same groups. Expression analysis based on the transcriptome data demonstrated that some CsGLP genes are preferentially expressed in specific tissues and may participate in specific tissue and organ development. In addition, the expression of some CsGLP genes was significantly changed under DM inoculation, as well as in response to salt, drought and ABA treatments, suggesting that CsGLP genes may play key roles in the response of cucumber to DM infection and various abiotic stresses. Our findings may lay a solid foundation for studying the functions of CsGLP genes in the future.

Supplementary Materials: The following are available online at https: / www.mdpi.com/article / 10.3390/horticulturae7100412/s1—Table S1: The gene-specific primers used for qRT-PCR, Table S2: The accession numbers of GLPs for phylogenetic analysis.

Author Contributions: Data curation, L.L. and Y.Z.; Funding acquisition, S.L. and Y.Z.; Investigation, Z.H., Y.Y. and Y.Z.; Methodology, L.L.; Resources, Z.H., S.L. and Y.Y.; Software, L.L. and Z.H.; Writing—original draft, S.L., Y.Y. and Y.Z.; Writing-review \& editing, Y.Y. and Y.Z. All authors have read and agreed to the published version of the manuscript.

Funding: This work was funded by the Academic and Technical Leader Plan of Jiangxi Provincial Main Disciplines [grant number 20204BCJ22023], the Natural Science Foundation of Jiangxi Province, China [grant number 20202BABL205002], the Science and Technology Project of Jiangxi Provincial Department of Education [grant number GJJ200464], and the National Natural Science Foundation of China [grant numbers 31860566 and 32160709].

Institutional Review Board Statement: Not applicable.

Informed Consent Statement: Not applicable.

Data Availability Statement: Not applicable.

Conflicts of Interest: The authors declare that they have no conflict of interest.

\section{References}

1. Lane, B.G.; Cuming, A.C.; Frégeau, J.; Carpita, N.C.; Hurkman, W.J.; Bernier, F.; Dratewka-Kos, E.; Kennedy, T.D. Germin isoforms are discrete temporal markers of wheat development. Eur. J. Biochem. 1992, 209, 961-969. [CrossRef] [PubMed]

2. Thompson, E.W.; Lane, B.G. Relation of protein synthesis in imbibing wheat embryos to the cell-free translational capacities of bulk mRNA from dry and imbibing embryos. J. Biol. Chem. 1980, 255, 5965-5970. [CrossRef]

3. Patnaik, D.; Khurana, P. Germins and germin like proteins: An overview. Indian J. Exp. Biol. 2001, 39, 191-200. [PubMed]

4. Dunwell, J.M.; Gibbings, J.G.; Mahmood, T.; Saqlan Naqvi, S.M. Germin and germin-like proteins: Evolution, structure, and function. Crit. Rev. Plant Sci. 2008, 27, 342-375. [CrossRef]

5. Ayub, S.; Hayat, R.; Zainab, Z.; Akhtar, W.; Mahmood, T. OsRGLP2 promoter derived GUS expression in transgenic tobacco in response to salicylic acid, $\mathrm{H}_{2} \mathrm{O}_{2}, \mathrm{PEG}, \mathrm{NaCl}$ and auxins. Plant Gene 2019, 19, 100190. [CrossRef]

6. Li, L.; Xu, X.; Chen, C.; Shen, Z. Genome-wide characterization and expression analysis of the germin-like protein family in rice and Arabidopsis. Int. J. Mol. Sci. 2016, 17, 1622. [CrossRef]

7. Breen, J.; Bellgard, M. Germin-like proteins (GLPs) in cereal genomes: Gene clustering and dynamic roles in plant defence. Funct. Integr. Genom. 2010, 10, 463-476. [CrossRef]

8. Ahmad, A.; Ahmed, A.; Essa, R.; Baber, S.; Jamshed, A.; Shoukat, A.; Younas, T.; Saleem, M.A.; Naveed, M. In-silico analysis of grapevine germin like protein (VvGLP3) and its probable role in defense against powdery mildew disease. Life Sci. J. Pak. 2019, 1, $17-23$. 
9. Barman, A.R.; Banerjee, J. Versatility of germin-like proteins in their sequences, expressions, and functions. Funct. Integr. Genom. 2015, 15, 533-548. [CrossRef] [PubMed]

10. Pei, Y.; Zhu, Y.; Jia, Y.; Ge, X.; Li, X.; Li, F.; Hou, Y. Molecular evidence for the involvement of cotton GhGLP2, in enhanced resistance to Verticillium and Fusarium Wilts and oxidative stress. Sci. Rep. 2020, 10, 12510. [CrossRef] [PubMed]

11. Beracochea, V.C.; Almasia, N.I.; Peluffo, L.; Nahirñak, V.; Hopp, E.H.; Paniego, N.; Heinz, R.A.; Vazquez-Rovere, C.; Lia, V.V. Sunflower germin-like protein HaGLP1 promotes ROS accumulation and enhances protection against fungal pathogens in transgenic Arabidopsis thaliana. Plant Cell Rep. 2015, 34, 1717-1733. [CrossRef]

12. Rodríguez-López, M.; Baroja-Fernández, E.; Zandueta-Criado, A.; Moreno-Bruna, B.; Muñoz, F.J.; Akazawa, T.; Pozueta-Romero, J. Two isoforms of a nucleotide-sugar pyrophosphatase/phosphodiesterase from barley leaves (Hordeum vulgare L.) are distinct oligomers of HvGLP1, a germin-like protein. FEBS Lett. 2001, 490, 44-48. [CrossRef]

13. Huang, N.; Cheng, X.; Hu, W.; Pan, S. Inactivation, aggregation, secondary and tertiary structural changes of germin-like protein in Satsuma mandarine with high polyphenol oxidase activity induced by ultrasonic processing. Biophys. Chem. 2015, 197, 18-24. [CrossRef] [PubMed]

14. Cheng, X.; Huang, X.; Liu, S.; Tang, M.; Hu, W.; Pan, S. Characterization of germin-like protein with polyphenol oxidase activity from Satsuma mandarine. Biochem. Biophys. Res. Commun. 2014, 449, 313-318. [CrossRef] [PubMed]

15. Banerjee, J.; Maiti, M.K. Functional role of rice germin-like protein1 in regulation of plant height and disease resistance. Biochem. Biophys. Res. Commun. 2010, 394, 178-183. [CrossRef] [PubMed]

16. Wang, H.; Zhang, Y.; Xiao, N.; Zhang, G.; Wang, F.; Chen, X.; Fang, R. Rice GERMIN-LIKE PROTEIN 2-1 functions in seed dormancy under the control of abscisic acid and gibberellic acid signaling pathways. Plant Physiol. 2020, 183, 1157-1170. [CrossRef]

17. Perveen, S.; Khan, T.A.; Shaheen, H.; Naz, R.; Hyder, M.Z.; Ijaz, B.; Naqvi, S.M.S.; Yasmin, T. Morpho-physiological investigations in transgenic tomato (Solanum lycopersicum L.) over expressing OsRGLP1 gene. Vitr. Cell Dev. Biol.-Plant 2021. [CrossRef]

18. Fu, J.Y.; Wang, X.C.; Mao, T.F.; Cheng, H.; Chen, F.; Yang, Y.J. Identification and functional analysis of germin-like protein gene family in tea plant (Camellia sinensis). Sci. Hortic. 2018, 234, 166-175. [CrossRef]

19. Das, A.; Pramanik, K.; Sharma, R.; Gantait, S.; Banerjee, J. In-silico study of biotic and abiotic stress-related transcription factor binding sites in the promoter regions of rice germin-like protein genes. PLoS ONE 2019, 14, e0211887. [CrossRef] [PubMed]

20. Banerjee, J.; Gantait, S.; Maiti, M.K. Physiological role of rice germin-like protein 1 (OsGLP1) at early stages of growth and development in indica rice cultivar under salt stress condition. Plant Cell Tissue Organ Cult. 2017, 131, 127-137. [CrossRef]

21. He, Z.D.; Tao, M.L.; Leung, D.W.M.; Yan, X.Y.; Chen, L.; Peng, X.X.; Liu, E.E. The rice germin-like protein OsGLP1 participates in acclimation to UV-B radiation. Plant Physiol. 2021, 186, 1254-1268. [CrossRef] [PubMed]

22. Zhang, N.; Guan, R.; Yang, Y.; Bai, Z.; Ge, F.; Liu, D. Isolation and characterization of a Fusarium oxysporum-resistant gene LrGLP1 from Lilium regale Wilson. In Vitro Cell Dev. Biol.-Plant 2017, 53, 461-468. [CrossRef]

23. Majeed, N.; Hussain, I.; Abbas, Z.; Iqbal, U.; Sultana, T.; Naqvi, S.M.S. Heterologous expression of OsRGLP1 gene induces resistance against fusarium infection in transgenic potato. Pak. J. Phytopathol. 2016, 28, 45-55.

24. Sultana, T.; Deeba, F.; Naz, F.; Rose, R.J.; Saqlan Naqvi, S.M. Expression of a rice GLP in Medicago truncatula exerting pleiotropic effects on resistance against Fusarium oxysporum through enhancing FeSOD-like activity. Acta Physiol. Plant. 2016, $38,255$. [CrossRef]

25. Liu, Q.; Yang, J.; Yan, S.; Zhang, S.; Zhao, J.; Wang, W.; Yang, T.; Wang, X.; Mao, X.; Dong, J.; et al. The germin-like protein OsGLP2-1 enhances resistance to fungal blast and bacterial blight in rice. Plant Mol. Biol. 2016, 92, 411-423. [CrossRef] [PubMed]

26. Zhang, Y.; Wang, X.; Chang, X.; Sun, M.; Zhang, Y.; Li, W.; Li, Y. Overexpression of germin-like protein GmGLP10 enhances resistance to Sclerotinia sclerotiorum in transgenic tobacco. Biochem. Biophys. Res. Commun. 2018, 497, 160-166. [CrossRef] [PubMed]

27. Pei, Y.; Li, X.; Zhu, Y.; Ge, X.; Sun, Y.; Liu, N.; Jia, Y.; Li, F.; Hou, Y. GhABP19, a novel germin-like protein from Gossypium hirsutum, plays an important role in the regulation of resistance to verticillium and fusarium wilt pathogens. Front. Plant Sci. 2019, 10, 583. [CrossRef] [PubMed]

28. Nakata, M.; Watanabe, Y.; Sakurai, Y.; Hashimoto, Y.; Matsuzaki, M.; Takahashi, Y.; Satoh, T. Germin-like protein gene family of a moss, Physcomitrella patens, phylogenetically falls into two characteristic new clades. Plant Mol. Biol. 2004, 56, 381-395. [CrossRef] [PubMed]

29. Lu, M.; Han, Y.P.; Gao, J.G.; Wang, X.J.; Li, W.B. Identification and analysis of the germin-like gene family in soybean. BMC Genom. 2010, 11, 620. [CrossRef] [PubMed]

30. Wang, X.; Zhang, H.; Gao, Y.; Sun, G.; Zhang, W.; Qiu, L. A comprehensive analysis of the Cupin gene family in soybean (Glycine max). PLoS ONE 2014, 9, e110092. [CrossRef]

31. Yuan, B.; Yang, Y.; Fan, P.; Liu, J.; Xing, H.; Liu, Y.; Feng, D. Genome-wide identification and characterization of germin and germin-like proteins (GLPs) and their response under powdery mildew stress in wheat (Triticum aestivum L.). Plant Mol. Biol. Rep. 2021. [CrossRef]

32. Lai, W.; Zhou, Y.; Pan, R.; Liao, L.; He, J.; Liu, H.; Yang, Y.; Liu, S. Identification and expression analysis of stress-associated proteins (SAPs) containing A20/AN1 zinc finger in cucumber. Plants 2020, 9, 400. [CrossRef]

33. Lai, W.; Zhu, C.; Hu, Z.; Liu, S.; Wu, H.; Zhou, Y. Identification and transcriptional analysis of zinc finger-homeodomain (ZF-HD) family genes in cucumber. Biochem. Genet. 2021, 59, 884-901. [CrossRef] 
34. Li, Z.; Zhang, Z.; Yan, P.; Huang, S.; Fei, Z.; Lin, K. RNA-Seq improves annotation of protein-coding genes in the cucumber genome. BMC Genom. 2011, 12, 540. [CrossRef]

35. Adhikari, B.N.; Savory, E.A.; Vaillancourt, B.; Childs, K.L.; Hamilton, J.P.; Day, B.; Buell, C.R. Expression profiling of Cucumis sativus in response to infection by Pseudoperonospora cubensis. PLoS ONE 2012, 7, e34954. [CrossRef] [PubMed]

36. Chen, C.; Chen, H.; Zhang, Y.; Thomas, H.R.; Frank, M.H.; He, Y.; Xia, R. TBtools: An integrative toolkit developed for interactive analyses of big biological data. Mol. Plant 2020, 13, 1194-1202. [CrossRef] [PubMed]

37. Gu, R.; Song, X.; Liu, X.; Yan, L.; Zhou, Z.; Zhang, X. Genome-wide analysis of CsWOX transcription factor gene family in cucumber (Cucumis sativus L.). Sci. Rep. 2020, 10, 6216. [CrossRef]

38. Zhou, Y.; Li, G.; Zhang, L.; Xu, J.; Hu, L.; Jiang, L.; Liu, S. Comprehensive genomic analysis and expression profiling of the BTB and TAZ (BT) genes in cucumber (Cucumis sativus L.). Czech J. Genet. Plant Breed. 2020, 56, 15-23. [CrossRef]

39. Muhammad, I.; Muhammad, I.; Tariq, M.; Hazrat, H.; Latif-ur, R.; Ijaz, N.; Khaliq-ur, R. Analysis of germin-like protein genes (OsGLPs) family in rice using various in silico approaches. Curr. Bioinform. 2020, 15, 17-33.

40. Sun, M.; Ye, Z.; Tan, J.; Chen, S.; Zhang, X.; Tu, L. A cotton germin-like protein GbGLP2 controls fiber length via regulating genes involved in secondary cell wall synthesis. Mol. Breed. 2020, 40, 98. [CrossRef]

41. Sun, C.; Song, X.; Zheng, J.; Li, X.; Feng, Z.; Yan, L. Comparative proteomic profiles of resistant/susceptible cucumber leaves in response to downy mildew infection. Hortic. Plant J. 2021, 7, 327-340. [CrossRef]

42. Gao, X.; Guo, P.; Wang, Z.; Chen, C.; Ren, Z. Transcriptome profiling reveals response genes for downy mildew resistance in cucumber. Planta 2021, 253, 112. [CrossRef] [PubMed]

43. Manosalva, P.M.; Davidson, R.M.; Liu, B.; Zhu, X.; Hulbert, S.H.; Leung, H.; Leach, J.E. A germin-like protein gene family functions as a complex quantitative trait locus conferring broad-spectrum disease resistance in rice. Plant Physiol. 2009, 149, 286-296. [CrossRef] [PubMed]

44. Giarola, V.; Chen, P.; Dulitz, S.J.; König, M.; Manduzio, S.; Bartels, D. The dehydration- and ABA-inducible germin-like protein CPGLP1 from Craterostigma plantagineum has SOD activity and may contribute to cell wall integrity during desiccation. Planta 2020, 252, 84. [CrossRef] [PubMed] 